\title{
ORIGINAL ARTICLE \\ The role of phenotypic plasticity on population differentiation
}

\begin{abstract}
M Schmid and F Guillaume
Several evolutionary processes shape the genetic and phenotypic differentiation of populations. Among them, the joint effects of gene flow, selection and phenotypic plasticity are poorly known, especially when trying to understand how maladaptive plasticity affects population divergence. We extended a quantitative genetic model of Hendry et al. (2001) to describe these joint effects on phenotypic and additive genetic divergence between two populations, and their phenotypic and genetic differentiation $\left(P_{\mathrm{ST}}\right.$ and $\left.Q_{\mathrm{ST}}\right)$. With individual-based simulations, we tested our model predictions and further modeled allelic differentiation at neutral $\left(F_{S T}\right)$ and adaptive $\left(F_{\text {STQ }}\right)$ loci. While adaptive phenotypic plasticity allows for large phenotypic divergence and differentiation despite high gene flow, maladaptive plasticity promotes genetic divergence and generates countergradient variation, under extensive migration with phenotypic differences sometimes opposed to genetic differences. Maladaptive plasticity can also promote adaptive phenotypic divergence by reducing the effective gene flow. Overall, plasticity decouples genetic from phenotypic differences between populations, and blurs the correlation between phenotypic divergence and local adaptation. By deriving models of population differentiation for three different life cycles, we further describe the effect of a species' ecology on evolution in structured populations.
\end{abstract}

Heredity (2017) 119, 214-225; doi:10.1038/hdy.2017.36; published online 26 July 2017

\section{INTRODUCTION}

Most species inhabit heterogeneous environments to which they adapt. As a result, species often show gradual changes in morphologies, behaviors or genetic composition across their range, which often coincide with environmental variation. In our current view of adaption as the result of character optimization by natural selection, the degree of adaptation of a local population is defined by how close its mean phenotypic value is to its local optimum. Many evolutionary processes are involved in making the population sit more or less close to its adaptive peak. Besides selection, pulling the population toward its peak, mutation, drift and migration will mainly cause deviations away from it (Lenormand, 2002; Blanquart et al., 2012). In particular, gene flow, mediated by migration between divergent habitats, will decrease population phenotypic divergence and limit local adaptation by bringing in locally maladapted alleles and reducing mean population fitness, which is described as migration load. If too strong, gene flow may eventually cause local adaptation to collapse by homogenizing allele frequencies across populations. Nevertheless, under migration-selection balance, an equilibrium phenotypic divergence may be maintained that is smaller than the environmental divergence (Slatkin, 1987; García-Ramos and Kirkpatrick, 1997; Hendry et al., 2001; Lenormand, 2002; Huisman and Tufto, 2012), and, for instance, may cause phenotypes to vary more gradually across space than the environment does (Kirkpatrick and Barton, 1997). The population phenotypic differentiation that ensues is the result of the differentiation of allele frequencies between populations. However, if the phenotype is directly influenced by the environment, genetic and phenotypic differentiations can be decoupled (Crispo, 2008).

A phenotype is plastic and, although genetically determined, is also directly influenced by the environment. Phenotypic plasticity has long been recognized as a ubiquitous property of organisms, whereby a single genotype may express different phenotypes in different environments (Woltereck, 1909; Bradshaw, 1965; Scheiner, 1993). Whether plasticity aids adaptive evolution depends on how it improves the fitness of individuals. A plastic response is said to be 'adaptive' when it allows genotypes to express phenotypes more closer to the environmental optimum, and it is called 'maladaptive' otherwise (Bradshaw, 1965; Stearns, 1989; Ghalambor et al., 2007; Hendry, 2016). Numerous examples of adaptive and maladaptive plasticity exist in nature. For instance, freshwater snails (Radix balthica) exhibit a high diversity in shell coloration within their distribution range. Ahlgren et al. (2013) showed that these differences could be explained (at least partially) by adaptive plastic responses to heterogeneous environments as snails respond to predator cues and ultraviolet light by changes in shell pigmentation. Similarly, metabolic rates in ectotherms are tightly linked to ambient temperatures, and individuals transferred to lower temperatures (for example, higher latitudes and altitudes) show a decreased growth rate. However, colder environments often have shorter growing seasons and require a faster growth to complete the life cycle within the growing season, which makes the plastic response maladaptive (Merilä et al., 2000). Nonetheless, populations of ectotherms in cold environments often exhibit similar or even higher growth rates compared to ectotherms in warmer habitats by

Department of Evolutionary Biology and Environmental Studies, University of Zurich, Zurich, Switzerland

Correspondence: Professor F Guillaume, Department of Evolutionary Biology and Environmental Studies, University of Zurich, Winterthurerstrasse 190, CH-8057 Zurich, Switzerland.

E-mail: frederic.guillaume@ieu.uzh.ch

Received 22 February 2017; revised 13 April 2017; accepted 19 May 2017; published online 26 July 2017 
genetically compensating for the maladaptive plastic response (Conover and Present, 1990). By this process of genetic compensation, patterns of countergradient variation arise when genetic differences are opposite to environmental differences between populations, which received broad attention in the field of ecology and evolution (Levins, 1969; Conover and Schultz, 1995; Grether, 2005). Examples of countergradient variation have been repeatedly revealed in common garden and reciprocal transplant experiments (Conover et al., 2009) and highlight that phenotypic plasticity can decouple the phenotypic from the genetic divergence observed between populations.

Although these effects of phenotypic plasticity have been recognized early on in evolutionary biology (Woltereck, 1909; Johannsen, 1911; Bradshaw, 1965; Levins, 1969), the implications of a break in correspondence between genetic and phenotypic divergence are as relevant as ever. It is especially genetic divergence that receives broad attention at the present time. Experimental approaches and molecular genetic methods now enable to pinpoint the molecular basis of local adaptation, ecological diversification and speciation (Whitlock, 2008; Seehausen et al., 2014; Rellstab et al., 2015; Whitlock and Lotterhos, 2015) and link ecological with evolutionary processes (Pelletier et al., 2009). However, genetic divergence is not guaranteed to cause phenotypic differentiation if resulting from genetic compensation (Grether, 2005) or may even be favored by adaptive plasticity depending on the timing of development relative to selection and dispersal (Thibert-Plante and Hendry, 2011). Thus, a full understanding of evolution in structured populations requires to also consider phenotypic plasticity because genetic divergence and phenotypic plasticity are alternative solutions to achieve trait divergence, and they not only cooccur but interact with each other (Schlichting and Pigliucci, 1998). While empirical studies often reveal possible outcomes of such interactions and contribute to a better understanding of genetic evolution (Dayan et al., 2015; Ghalambor et al., 2015), a quantitative theory is missing, but necessary to better weight the contributions of potential causative factors (Crispo, 2008). Most theoretical studies of phenotypic plasticity in heterogeneous environments have focused on the evolution of plasticity itself (Via and Lande, 1985; Sultan and Spencer, 2002; Chevin and Lande, 2011) rather than trying to quantify the effect of plasticity on genetic and phenotypic differentiation per se (but see Crispo, 2008, for a conceptual model). Moreover, few studies consider maladaptive plasticity in the context of adaptation with gene flow despite it being considered widespread in nature (Crispo, 2008; Hendry, 2016) and potentially playing a key role in the evolution of population adaptive divergence (for example, Ghalambor et al., 2015).

Our goal is to quantify the effect of plasticity on genetic and phenotypic differentiation and divergence between populations adapted to divergent habitats. To this end, we extend a simple twopatch quantitative genetic model (Hendry et al., 2001) that describes the combined effect of selection, gene flow and genetic variance on phenotypic divergence. We incorporate phenotypic plasticity in this model as a two-parameter reaction norm (intercept and slope) and derive phenotypic divergence between the two patches at migrationselection equilibrium. We also derive simple formulas for indices of phenotypic and genetic differentiation ( $P_{\mathrm{ST}}$ and $Q_{\mathrm{ST}}$ ), and use individual-based simulations to estimate the effect of phenotypic plasticity on population differentiation at neutral $\left(F_{\mathrm{ST}}\right)$ and adaptive $\left(F_{\mathrm{STQ}}\right)$ loci. Differentiation indices, either at the trait or the locus level, are important tools widely used to assess the degree of local adaptation in natural populations or to detect loci under selection (Lewontin and Krakauer, 1973; Spitze, 1993). In accordance with the verbal model of Crispo (2008), we show analytically how adaptive phenotypic plasticity directs phenotypic divergence and differentiation toward the trait

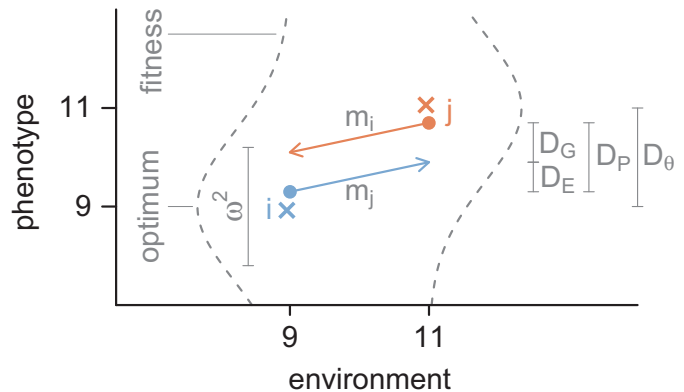

Figure 1 Two populations $(i, j)$ inhabit different environments and consequently exhibit different local phenotypic optima (indicated by the crosses in orange and blue). Gene flow may cause a deviation of the average phenotypic value of each population (orange and blue circles) from their trait optima. A Gaussian function describes the fitness of different phenotypes in the respective population (dotted lines) with the variance $\left(\omega^{2}\right)$ determining the local selection strength. Gene flow between the two populations (arrows) moves genotypes to the other population at specific rates $\left(m_{j}\right.$ and $m_{i}$ correspond to backward migration rates). Because of phenotypic plasticity, a change in the environment causes a change in the expressed phenotype $\left(D_{E}=g_{1}\left(e_{j}-e_{j}\right)\right)$. The degree of phenotypic plasticity $\left(g_{1}\right)$ is assumed to be constant in space and time. While $D_{P}$ is indicating the difference between average phenotypes of both populations $\left(D_{P}=z_{j}-z_{i}\right), D_{G}$ stands for genetic divergence $\left(D_{G}=g_{0 j}-g_{0}\right)$. The parameter $D_{\theta}$ represents the difference between the local phenotypic optima $\left(D_{\theta}=\theta_{j}-\theta_{j}\right)$.

optima while reducing genetic differences between populations. Maladaptive phenotypic plasticity has mostly opposite effects, favoring genetic differences between populations while reducing phenotypic differences. It also has the counterintuitive effect of inducing larger phenotypic divergence than with partially adaptive plasticity for some parameter values by reducing the effective migration rate between divergent populations. Finally, our approach highlights the consequences of variation in a species' ecology by modeling different timings of events in its life cycle.

\section{MATERIALS AND METHODS}

\section{Mathematical models}

The quantitative genetic model of Hendry et al. (2001) predicts the difference between the average phenotypes $\left(D_{P}\right)$ of two populations ( $i$ and $j$ ) at migrationselection equilibrium under weak selection. These two populations differ in their local phenotypic optima $\left(\Theta_{i}, \Theta_{j}\right)$ underlain by an environmental difference $\left(e_{i}, e_{j}\right)$. Gene flow between the two populations $\left(m_{i}, m_{j}\right)$ counteracts divergent selection and transfers 'maladapted' genotypes to the other population (Figure 1). We model symmetrical migration rates $\left(m_{i}=m_{j}\right)$, constant strength of selection and equal population sizes, for a species with discrete, nonoverlapping generations similar to the original model. To facilitate a direct comparison between the Hendry et al. (2001) model and our extension, we adopt many of their notations, while using the term migration instead of mixing.

We extended the model of Hendry et al. (2001) by adding plasticity in the form of a linear norm of reaction (NoR), where the phenotype $(z)$ of an individual is expressed in dependence of the genotype ( $g_{0}$-intercept, $g_{1}$ slope), the environment $(e)$ and a normally distributed residual environmental component within populations $\left(\varepsilon=N\left(0, \sqrt{V_{\epsilon}}\right)\right)$.

$$
z=g_{0}+g_{1} \cdot e+\varepsilon
$$

For all investigated scenarios, we assume that the degree of phenotypic plasticity is identical in both populations $\left(g_{1 i}=g_{1 j}=g_{1}\right)$ and constant over time. Importantly, we consider the evolution of phenotypic plasticity to be at an equilibrium state that is caused by a balance between costs and benefits of plastic responses (Via and Lande, 1985; Lande, 2009; Scheiner and Holt, 2012), physiological or genetic constraints (Langerhans and DeWitt, 2002) and by correlations between environmental variables (Chevin and Lande, 2015). We thereby do not rule out the possibility of evolving phenotypic plasticity 
a

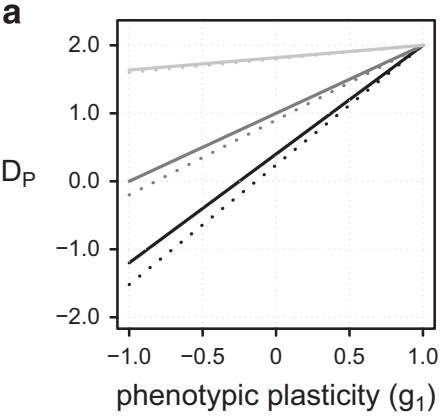

c

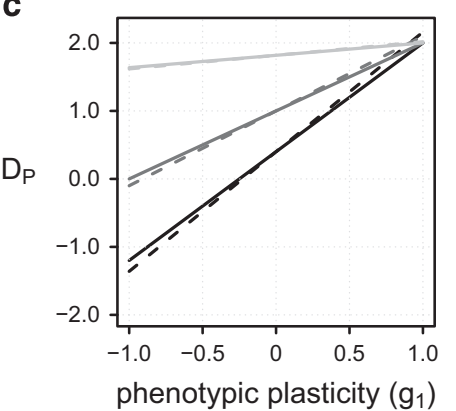

b
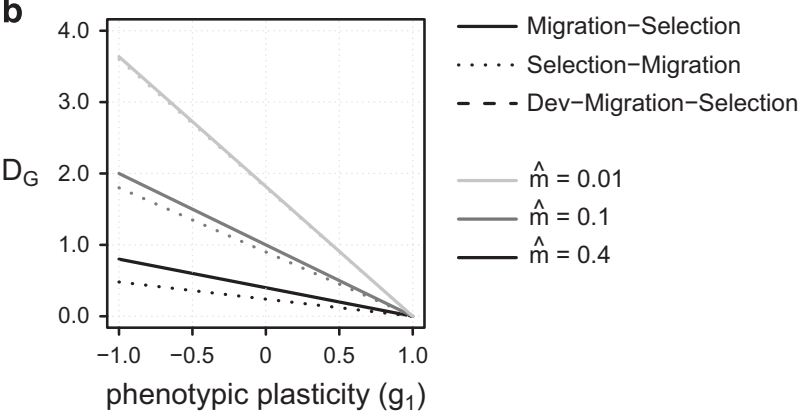

$\hat{m}=0.01$

$\hat{\mathrm{m}}=0.1$

$\hat{\mathrm{m}}=0.4$

d

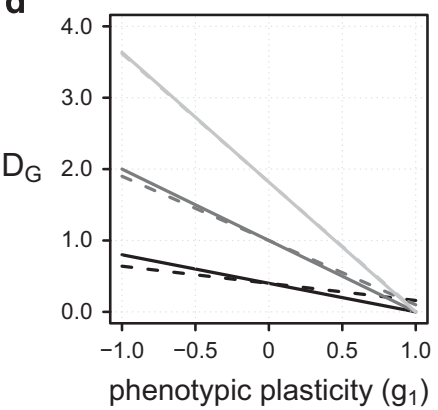

Figure 2 Phenotypic divergence $D_{P}^{*}\left(\mathbf{a}\right.$ and $\mathbf{c}$ ) and genetic divergence $D_{G}^{*}$ ( $\mathbf{b}$ and $\left.\mathbf{d}\right)$ predicted by our models, as a function of phenotypic plasticity $\left(g_{1}\right)$ in the three life cycles: Migration-Selection (solid lines), Selection-Migration (dotted lines) and Development-Migration-Selection (dashed lines). Results are for phenotypic and additive genetic variance $P_{w}=G_{w}=0.5$, selection strength $\omega^{2}=5$, effective migration rates $\hat{m}=0.0002$ (light gray), $\hat{m}=0.1$ (medium gray), $\hat{m}=0.8$ (dark gray) and a difference between the local phenotypic optima $D_{\Theta}=2$. Phenotypic plasticity $g_{1}$ together with an environmental distance $e_{j}-e_{j}=2$ refers to $-2<D_{E}<+2$.

but just do not describe explicitly the causes for specific equilibrium levels of plasticity.

\section{Phenotypic and genetic divergence (Migration-Selection)}

In the first model, dispersal, and thus migration, occurs in each population before selection. Extending the original Hendry et al. (2001) model, the average trait values after migration $\left(\bar{z}_{i}^{m}, \bar{z}_{j}^{m}\right)$ are derived from the initial mean trait values in population $i$ and $j\left(\bar{z}_{i}, \bar{z}_{j}\right.$, see Equation (1)) and the proportion of immigrants from the other population $\left(m_{i}, m_{j}\right)$ :

$$
\begin{aligned}
& \bar{z}_{i}^{m}=m_{i}\left(\bar{g}_{0 j}+\bar{g}_{1} e_{i}\right)+\left(1-m_{i}\right)\left(\bar{g}_{0 i}+\bar{g}_{1} e_{i}\right), \\
& \bar{z}_{j}^{m}=m_{j}\left(\bar{g}_{0 i}+\bar{g}_{1} e_{j}\right)+\left(1-m_{j}\right)\left(\bar{g}_{0 j}+\bar{g}_{1} e_{j}\right) .
\end{aligned}
$$

The migration rates $m_{i}$ and $m_{i}$ are independent of population characteristics (for example, population density or the phenotypes or genotypes of migrants) and represent effective migration rates. Effective migration rates deviate from census migration rates $\left(m_{c}, m_{c j}\right)$ when migrants differ from residents in their fitness (compare Supplementary Figures S1a-h with Supplementary Figures S2a-d). The phenotype of migrating genotypes is expressed in the new environment after migration. This type of plastic response could either refer to developmental phenotypic plasticity, when individuals migrate prior to development (for example, seed dispersal; Beldade et al., 2011), or to labile phenotypic plasticity, when phenotypes can be adjusted multiple times during the life of an individual (West-Eberhard, 1989; Scheiner, 1993; Lande, 2014). Adding the effect of viability selection, the mean trait value after selection and reproduction $\left(z^{m s}\right)$ can be calculated from $z^{m}$, the additive genetic variance within populations $\left(G_{w}\right.$, the variance in $g_{0}$ within the respective population before selection) and the selection gradient $(\beta(z))$ :

$$
\begin{aligned}
& \bar{z}_{i}^{m s}=\bar{z}_{i}^{m}+G_{w i} \beta_{i}\left(\bar{z}_{i}^{m}\right) \\
& \bar{z}_{j}^{m s}=\bar{z}_{j}^{m}+G_{w j} \beta_{j}\left(\bar{z}_{j}^{m}\right) .
\end{aligned}
$$

As the migration rates are independent of population density and population fitness in these equations, viability selection corresponds to soft selection. Subsequent calculations of the change in mean trait value between generations $\left(\Delta \bar{z}=\bar{z}^{m s}-\bar{z}\right)$ and of the change in the difference between mean trait values $\left(\Delta D_{P}=\Delta z_{j}-\Delta z_{i}\right)$

$$
\Delta D_{P}=\left(m_{j}+m_{i}\right)\left(g_{0 i}-g_{0 j}\right)+G_{w j} \beta_{j}\left(\bar{z}_{j}^{m}\right)-G_{w i} \beta_{i}\left(\bar{z}_{i}^{m}\right),
$$

allow to solve for the difference between mean trait values $D_{P}=z_{j}-z_{i}=g_{0 j}+$ $g_{1} e_{j}-g_{0 i}-g_{1} e_{i}$ at equilibrium $\left(\Delta D_{P}=0\right)$ :

$$
D_{P}^{*}=g_{1}\left(e_{j}-e_{i}\right)+\left(\frac{1}{\hat{m}}\right)\left[G_{w j} \beta_{j}\left(\bar{z}_{j}^{m}\right)-G_{w i} \beta_{i}\left(\bar{z}_{i}^{m}\right)\right] .
$$

In all scenarios, the parameter $\hat{m}$ corresponds to the total effective migration rate $\hat{m}=m_{i}+m_{j}$. Assuming a Gaussian fitness function, weak selection and normally distributed phenotypes, the selection gradient becomes $\beta(\bar{z})=$ $-(\bar{z}-\Theta) /\left(\omega^{2}+P_{w}\right)$ (Via and Lande, 1985). The difference in mean trait values between populations at equilibrium $\left(D_{P}^{*}\right)$ is obtained by further assuming equal phenotypic $\left(P_{w i}=P_{w j}=P_{w}\right)$ and additive genetic $\left(G_{w i}=G_{w j}=G_{w}\right)$ variances within populations, and an environmental difference $\left(e_{j}-e_{i}\right)$ that corresponds to the difference between local phenotypic optima $\left(D_{\Theta}=\Theta_{j}-\Theta_{i}\right)$, such that the plastic change of the phenotype $D_{E}=g_{1}\left(e_{j}-e_{i}\right.$; Figure 1) can be expressed as $D_{E}=g_{1} D_{\Theta}$. The equilibrium divergence then is

$$
D_{P}^{*}=D_{\Theta} \frac{G_{w}+g_{1} \hat{m}\left(\varpi^{2}+P_{w}-G_{w}\right)}{G_{w}+\hat{m}\left(\varpi^{2}+P_{w}-G_{w}\right)} .
$$

Equation (6) shows that the equilibrium phenotypic divergence is a linear function of $g_{1}$, the extent of phenotypic plasticity (Figure 2a). It reduces to the expression of Hendry et al. (2001) when $g_{1}=0$. If plasticity is perfectly adaptive ( $\left.g_{1}=1\right)$, Equation (6) becomes $D_{P}=D_{\Theta}$; the two-population mean phenotypes are at their optimum irrespective of the amount of gene flow. In general, adaptive plasticity $\left(0<g_{1}<2\right.$ in our scenarios) reduces the effect of gene flow and shifts $D_{P}$ toward the optimal divergence $D_{\Theta}$ (Figure 2a). Note that hyperplasticity, which describes plastic responses in the direction of, but larger than perfect plasticity $\left(g_{1}>1\right.$; Scheiner and Holt, 2012), can be adaptive and 
can reduce the difference between $D_{P}$ and $D_{\Theta}\left(1<g_{1}<2\right)$. On the other hand, when plasticity is maladaptive $\left(g_{1}<0 ; g_{1}>+2\right)$, phenotypic divergence deviates more strongly from the optimal divergence and can even become negative when $\hat{m}$ is large (Figure 2a).

Based on the phenotypic divergence $\left(D_{P}^{*}\right)$ and the contribution of phenotypic plasticity, we find the additive genetic divergence $\left(D_{G}=g_{0 j}-g_{0 i}=z_{j}-z_{i}-g_{1}\right.$ $\left.\left(e_{j}-e_{i}\right)=D_{P}-D_{E}\right)$ at equilibrium as

$$
D_{G}^{*}=D_{\Theta} \frac{G_{w}\left(1-g_{1}\right)}{G_{w}+\hat{m}\left(\varpi^{2}+P_{w}-G_{w}\right)} .
$$

Therefore, adaptive plasticity reduces the genetic divergence of the populations to the point of no divergence $\left(D_{G}^{*}=0\right)$ when plasticity is perfect $\left(g_{1}=1\right)$, while maladaptive plasticity increases its extent (Figure 2b). Again, Equation (7) reduces to Equation (7) of Hendry et al. (2001) in the absence of plasticity $\left(g_{1}=0\right)$. More generally, our Equation (7) corresponds to Equation (7) in Hendry et al. (2001) when rescaling the environmental divergence $D_{\Theta}$ by plasticity $D_{\Theta}\left(1-g_{1}\right)$ in the latter. Finally, the link between $D_{P}^{*}$ and $D_{G}^{*}$ can simply be done using the following relationship: $D_{P}^{*}=D_{G}^{*}+g_{1} D_{\Theta}$. Phenotypic and genetic divergences are thus expected to be equivalent only in the absence of phenotypic plasticity.

As species may differ in the ability to respond plastically to novel environmental conditions and vary in the timing of development, we developed models for two alternative life cycles and provide the derivations in the Appendix. In the second life cycle, development (=phenotype expression) and viability selection occur prior to migration (Selection-Migration model), which can apply to species like damselflies or lepidopterans with rather long-lived developmental stages under selection and subsequent short-lived but dispersing adult stages. In the third model, development is followed by migration and then selection (Development-Migration-Selection model) such that individuals after migration do not respond plastically to the new environment. The third life cycle corresponds to species and traits with early phenotype determination (for example, by maternal effects) and rather late selection processes or to situations of a too slow plastic response to novel environmental conditions. While the general trends and patterns coincide across models (Figure 2), some systematic differences exist.

\section{Phenotypic and additive genetic differentiation}

The measures of genetic and phenotypic divergence seem to be obvious choices to quantify differences between populations, but they do not capture all aspects of population differentiation. Imagine two populations that exhibit a mean phenotypic divergence of five centimeters in body size, while individuals of the same population vary from each other with a variance of $10 \mathrm{~cm}$. Those two population are less differentiated than the same populations with a withinpopulation variance of only $1 \mathrm{~cm}$. Measures of differentiation $\left(P_{\mathrm{ST}}, Q_{\mathrm{ST}}, F_{\mathrm{ST}}\right.$ and $\left.F_{\mathrm{STQ}}\right)$ do account for both variance components, they can be directly compared to each other and are widely applied in biology.

Phenotypic differentiation $P_{\mathrm{ST}}$ represents the fraction of phenotypic variance between populations $\left(P_{b}\right)$ over the total phenotypic variance (the sum of $P_{b}$ and twice the within-population variance component $P_{w}$; Storz, 2002; Leinonen et al., 2006):

$$
P_{\text {ST }}=\frac{P_{b}}{P_{b}+2 P_{w}} .
$$

In a two-population setting, the phenotypic variance between populations at equilibrium can be derived from $D_{P}^{*}$ as

$$
P_{b}=\left(\frac{D_{P}^{*}}{2}\right)^{2}
$$

As we assume that individuals in the two populations differ only in their genetic $\left(g_{0}\right)$ and environmental values $\left(e_{j}, e_{i}\right)$, while no other factors contribute to phenotypic variance, phenotypic equals additive genetic variance within populations such that $P_{w}=G_{w}$. However, plasticity can increase the phenotypic variance $\left(P_{w}\right)$ above the genetic variance within populations $\left(G_{w}\right)$, as described previously (De Jong, 1990; Gavrilets and Scheiner, 1993). Resulting from variance in the NoR components $g_{0}, g_{1}, e$ and $\varepsilon$ (Equation (1)) within a population $\left(G_{w}, G_{w 1}, E\right.$ and $\left.V_{\varepsilon}\right)$, the effect of phenotypic plasticity on phenotypic variance within populations $P_{w}$ can be computed as

$$
\begin{aligned}
P_{w}= & G_{w}+\bar{g}_{1}^{2} E+\bar{e}^{2} G_{w 1}+G_{w 1} E+2 \operatorname{cov}\left(e, g_{1}\right)+2 \operatorname{cov}\left(e, g_{0}\right) \\
& +2 \operatorname{cov}\left(g_{0}, g_{1}\right)+V_{\epsilon}
\end{aligned}
$$

(Gavrilets and Scheiner, 1993). Consequently, phenotypic plasticity in combination with environmental variance within populations $(E>0)$ increases $P_{w}$ over $G_{w}$ while we recover $P_{w}=G_{w}+V_{\varepsilon}$ in the absence of plasticity $\left(g_{1}=0\right)$.

$Q_{S T}$ describes population genetic differentiation as the ratio of the additive genetic variance between populations $\left(G_{b}\right)$ over the total additive genetic variance (the sum of $G_{b}$ and twice the additive genetic variance within populations $G_{w}$; Spitze, 1993; Whitlock, 2008).

$$
Q_{\mathrm{ST}}=\frac{G_{b}}{G_{b}+2 G_{w}} .
$$

Following Equation (7), the genetic variance between populations $\left(G_{b}\right)$ at equilibrium can be derived from the additive genetic divergence $\left(D_{G}^{*}\right)$.

$$
G_{b}=\left(\frac{D_{G}^{*}}{2}\right)^{2}
$$

The within-population additive genetic variance is identical to the parameter $G_{w}$ used in the analytical models.

\section{Individual-based simulations}

In addition to the analytical model, we ran individual-based genetically explicit simulations with a modified version of Nemo2.3 (Guillaume and Rougemont, 2006). Individual-based simulations help uncover the dynamics of the additive genetic variance $G_{w}$ (a fixed parameter of the analytical model), and of the effective migration (a function of the realized phenotypic divergence of the populations), and allow to study additional aspects of population differentiation that are not addressed by the analytical model (differentiation in allele frequencies $F_{\mathrm{ST}}$ at neutral markers and $F_{\mathrm{STQ}}$ at quantitative trait loci).

In our simulations, each diploid individual carried $n=50$ unlinked additive loci. Mutations followed a continuum-of-alleles model with a zero-centered normal distribution of effects $N\left(0, \alpha^{2}=0.1\right)$ and a mutation rate of $\mu_{q}=0.0001$ (mutational variance: $V_{m}=2 \cdot n \cdot \mu_{q} \cdot \alpha^{2}=0.001$ ). In addition, each individual carried 50 unlinked neutral loci that followed a single-step mutation model with a mutation rate of $\mu_{n}=0.0001$ and a maximum of 256 alleles per locus. The neutral loci thus behaved like SSR markers (microsatellites). Phenotypic plasticity was implemented into Nemo as a linear NoR. The phenotype of each individual $\left(z_{\text {ind }}\right)$ was a function of its genetic contribution (intercept $g_{0}$ ), the degree of plasticity (slope $g_{1}$ ) and the environment $e: z_{\text {ind }}=g_{0}+g_{1} \cdot(e-10)$. The value 10 was an arbitrary offset value used to set an invariant point where NoR cross (Scheiner, 2013). The slope was set to a fixed value for all individuals, while the intercept was modeled as an additive polygenic quantitative trait subject to mutation, recombination and selection. A given genotype expressed phenotypes in population $i$ and $j$ with difference $D_{E}=g_{1}\left(e_{j}-e_{i}\right)=g_{1} D_{\Theta}$, as we set the phenotypic optima and the environmental values to $e_{i}=\Theta_{i}=9$ and $e_{j}=\Theta_{j}=11$, whereby $D_{\Theta}$ was 2 in all simulations (Figure 1).

The two populations had a carrying capacity of 1000 diploid individuals each with an even sex ratio. Diecious individuals mated at random without selfing within a population, and female fecundity was Poisson-distributed with a mean of 4. For each offspring produced by a female, a male was randomly chosen. The life cycle of each individual for the Migration-Selection model (MS) was as follows: breeding (when offspring were created) $\rightarrow$ migration (of the offspring) $\rightarrow$ phenotype expression (in dependence of the environment) $\rightarrow$ viability selection (on offspring) $\rightarrow$ population regulation (when the number of offspring was reduced to the carrying capacity, randomly with regard to the phenotype, and survivors were transferred into the adult life stage for the next generation). The life cycle for the Selection-Migration model (SM) was as follows: breeding $\rightarrow$ phenotype expression $\rightarrow$ viability selection $\rightarrow$ migration $\rightarrow$ population regulation. The life cycle for the Development-Migration-Selection model (DMS) was as follows: breeding $\rightarrow$ phenotype expression $\rightarrow$ migration $\rightarrow$ viability selection $\rightarrow$ population regulation.

To match the assumption of stabilizing selection of the quantitative genetics model, Gaussian selection was modeled with parameter $\omega^{2}$ as the width of the selection surface, which relates to the strength of selection as $1 / \omega^{2}$. We ran 840 parameter combinations for each life cycle. We used four different selection strengths $\left(\omega^{2}=1,5,10\right.$ and 50), 10 different census migration rates 

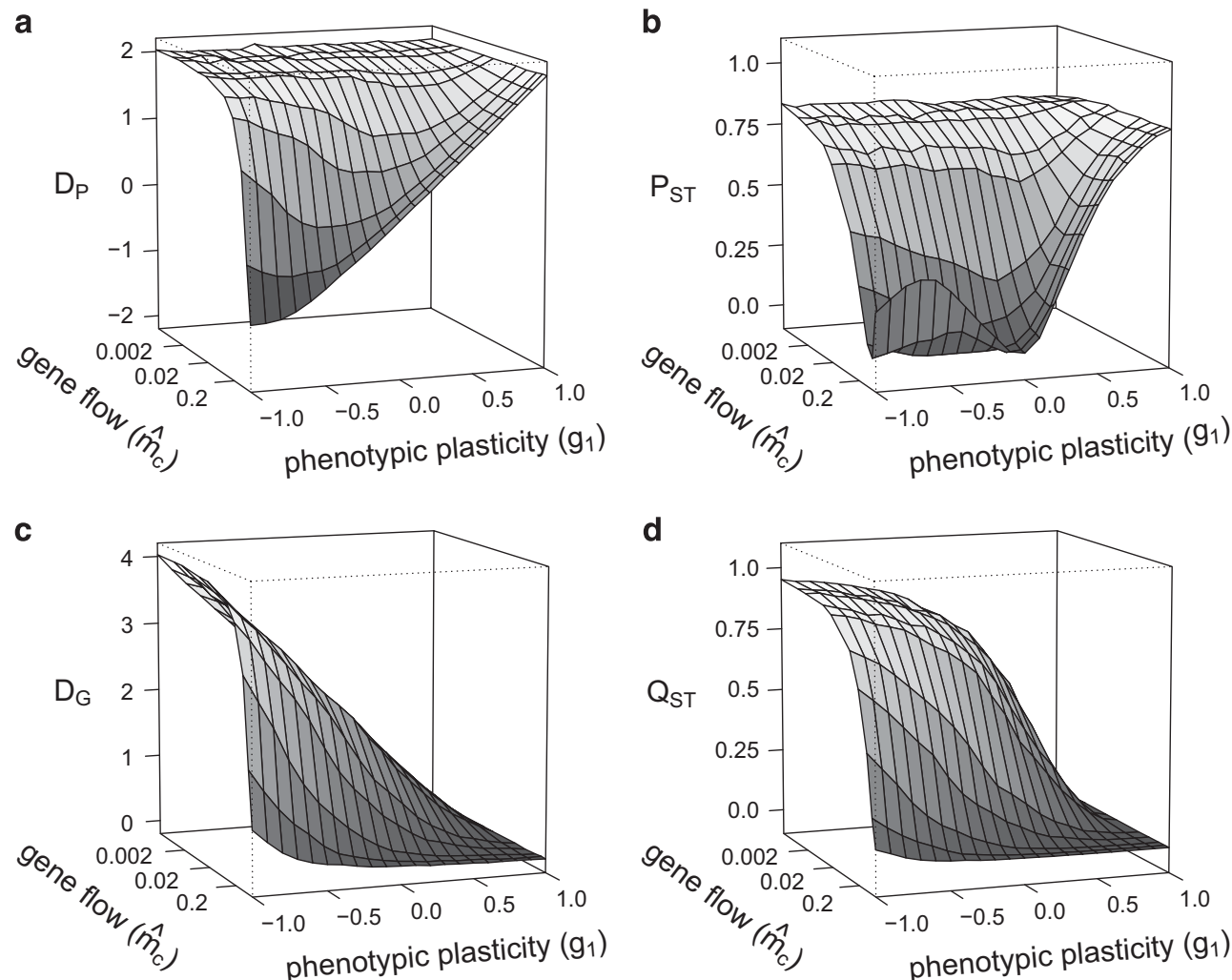

Figure 3 Simulation results with the MS life cycle for phenotypic divergence $D_{P}(\mathbf{a})$, phenotypic differentiation $P_{\mathrm{ST}}$ (b), additive genetic divergence $D_{G}$ (c) and differentiation in additive genetic effects $Q_{S T}$ (d) as function of phenotypic plasticity ( $g_{1}-$ NoR slope) and census gene flow ( $\hat{m}_{c}$; on a decimal logarithmic scale). Positive $g_{1}$ values indicate adaptive phenotypic plasticity, while negative values indicate maladaptive phenotypic plasticity. The graphs show the results for moderate selection strength $\left(\omega^{2}=5\right)$ after 100000 generations. The two populations differ in their local phenotypic optima by two units $\left(D_{\Theta}=+2\right)$, whereby both populations meet their local phenotypic optima at a phenotypic divergence of $D_{P}=+2$ (Figure 1 ).

$\left(m_{c i}=m_{c j}=0.0001,0.0005,0.001,0.005,0.01,0.05,0.1,0.2,0.3\right.$ and 0.4$)$ and 21 levels of phenotypic plasticity (the NoR slope values $g_{1}$ ranged from -1 to +1 , which corresponds to $D_{E}$ values ranging from -2 to +2 ). The census migration rate $\hat{m}_{c}$ corresponded to the rate of individuals migrating between populations, which did not necessarily correspond to the effective rate of individuals who migrated, survived and successfully reproduced $(\hat{m})$. As the difference between local phenotypic optima $D_{\Theta}$ was set to +2 in every scenario, all positive $g_{1}$ values represented adaptive phenotypic plasticity changing the phenotypic value of migrants toward the new local optimum. All negative $g_{1}$ values represented maladaptive phenotypic plasticity that pushed phenotypes farther away from the new local optimum. For each parameter combination, 20 replicates were run for 100000 generations to reach migration-selection equilibrium. After 100000 generations, the genotype tables of all adult individuals were exported.

Based on Equation (8), $P_{\mathrm{ST}}$ was calculated for the individual-based simulations by computing $P_{b}$ and $P_{w}$ from the genotype tables as follows:

$$
\begin{aligned}
& P_{b}=\frac{1}{2} \sum_{\text {pop } \in\{i, j\}}\left(\bar{z}_{\text {pop }}-\bar{z}_{\text {tot }}\right)^{2}, \\
& P_{w}=\frac{1}{2000} \sum_{\text {pop } \in\{i, j\}} \sum_{\text {ind }=1}^{1000}\left(z_{\text {ind }}-\bar{z}_{\text {pop }}\right)^{2},
\end{aligned}
$$

$Q_{\text {ST }}$ was calculated based on the additive genetic variance between populations and within populations (Equation (11)),

$$
\begin{aligned}
G_{b} & =\frac{1}{2} \sum_{\text {pop } \in\{i, j\}}\left(\bar{g}_{0 \text { pop }}-\bar{g}_{0 \text { tot }}\right)^{2}, \\
G_{w} & =\frac{1}{2000} \sum_{\text {pop } \in\{i, j\}} \sum_{\text {ind }=1}^{1000}\left(g_{0 \text { ind }}-\bar{g}_{0 \text { pop }}\right)^{2} .
\end{aligned}
$$

The differentiation in allele frequencies at neutral loci $F_{\mathrm{ST}}$ and at quantitative loci $F_{\text {STQ }}$ (Le Corre and Kremer, 2012) was calculated using the exported genotype tables for neutral and quantitative loci and the package hierfstat (Goudet, 2005) with the Weir-Cockerham $F_{\mathrm{ST}}$ estimate in R (R Development Core Team, 2008, version 3.2.3). For each parameter combination, the arithmetic mean overall 20 replicates for $D_{P}^{*}, D_{G}^{*}, P_{\mathrm{ST}}, Q_{\mathrm{ST}}, F_{\mathrm{ST}}$ and $F_{\mathrm{STQ}}$ was calculated.

To compare the individual-based simulations with the analytical models, we used the simulated values of $G_{w}, P_{w}$ and effective migration rates $\hat{m}$ (proportion of successfully reproducing immigrants) together with the parameter values of $D_{\Theta}, \omega^{2}$ and $g_{1}$ to compute the expected values of $D_{P}^{*}, D_{G}^{*}, P_{\mathrm{ST}}$ and $Q_{\mathrm{ST}}$ from the formulas derived above (Equations (5), (6), (8), (9), (10), (11) and (12)).

\section{RESULTS}

In the following sections, we refer exclusively to the results of the Migration-Selection models while treating the particularities of the other two life cycles (Selection-Migration and Development-Migration-Selection) separately.

\section{Effects of phenotypic plasticity on $D_{G}$ and $D_{P}$}

Overall, and as expected, adaptive phenotypic plasticity $\left(g_{1}>0\right)$ increased phenotypic divergence $D_{P}$ toward the optimal divergence of $D_{\Theta}=2$ despite gene flow (Figures 2a, c and 3a) and decreased genetic divergence $D_{G}$ (Figures $2 \mathrm{~b}, \mathrm{~d}$ and $3 \mathrm{c}$ ). Maladaptive phenotypic plasticity $\left(g_{1}<0\right)$ had the opposite effect, increasing the maladaptive effect of gene flow and leading to genetic divergence larger than the difference in trait optima (Figures 2b, d and 3c) and reduced phenotypic divergence, with $D_{P}$ even opposed to the difference in 
a

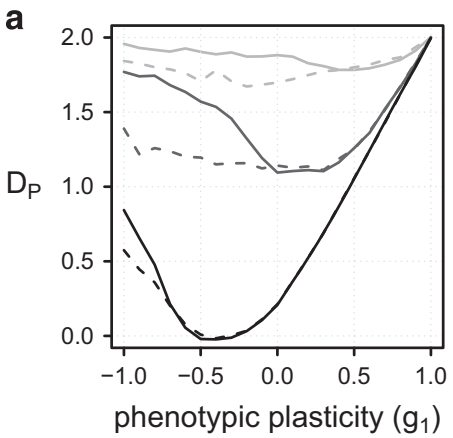

C

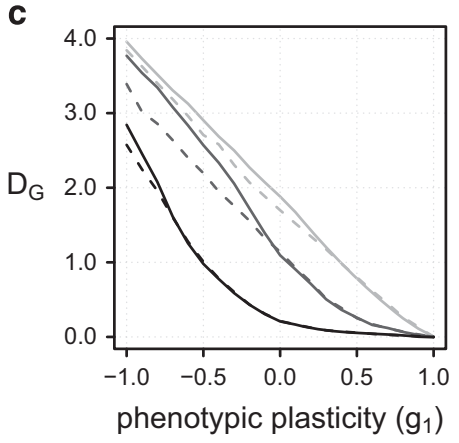

b

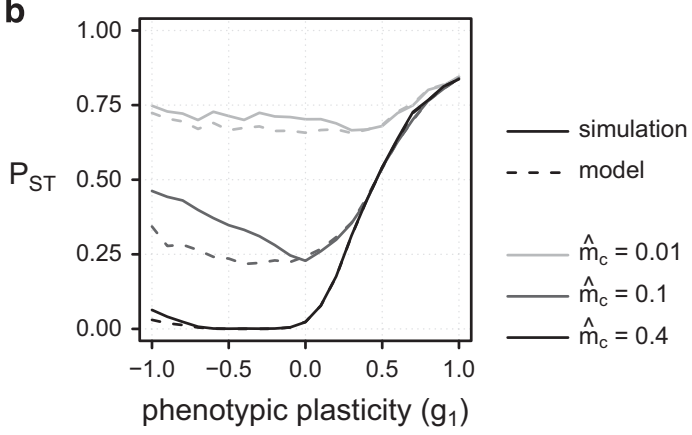

d

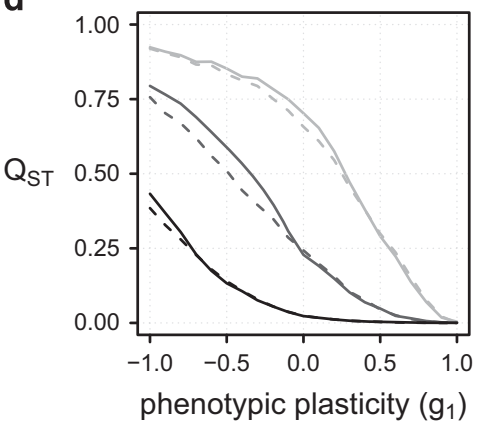

Figure 4 The four graphs illustrate the deviations between the individual-based simulations (solid lines) and the analytical predictions (dotted lines) for phenotypic and additive genetic divergence (a: $\left.D_{P} ; \mathbf{c}: D_{G}\right)$ and differentiation (b: $\left.P_{\mathrm{ST}} ; \mathbf{d}: Q_{\mathrm{ST}}\right)$ at moderate selection $\left(\omega^{2}=5\right)$ for three different census migration rates $\left(\hat{m}_{c}=0.4,0.1\right.$ and 0.01$)$. Note that the census migration rate may deviate considerably from the effective migration rate depending on phenotypic plasticity (Supplementary Figure S2). We used the effective migration rate to parameterize the MS model.

trait optima $\left(D_{P}<0\right)$ at high gene flow (Figures $2 \mathrm{a}, \mathrm{c}$ and $3 \mathrm{a}$; see also Supplementary Figures S3 and S4). Large maladaptive plasticity further led to the removal of immigrants with near-zero fitness under strong selection $\left(\omega^{2}=1\right)$, which caused an increase of phenotypic divergence at equilibrium because of a drastic reduction of effective gene flow (Figure 3a; Supplementary Figures S2 and S3).

Relations between phenotypic plasticity, $P_{\mathrm{ST}}$ and $Q_{\mathrm{ST}}$

The indices of phenotypic $\left(P_{\mathrm{ST}}\right)$ and genetic $\left(Q_{\mathrm{ST}}\right)$ differentiation closely followed the pattern of their respective divergence values; $P_{\mathrm{ST}}$ was large when $D_{P}$ was large (Equations (8) and (9)) and likewise for $Q_{S T}$ and $D_{G}$ (Equations (11) and (12)). In consequence, gene flow decreased $P_{\mathrm{ST}}$ for any level of phenotypic plasticity unless strong adaptive plasticity maintained divergence, or when maladaptive plasticity caused the divergence to cross zero and become negative, opposite to the trait optima (Figures $3 \mathrm{a}$ and b; Supplementary Figure S5). In contrast, $Q_{\mathrm{ST}}$ was always decreasing with gene flow and always larger for maladaptive than adaptive plasticity, in agreement with the pattern of genetic divergence (Figures $3 \mathrm{c}$ and $\mathrm{d}$; Supplementary Figure S6). Therefore, adaptive plasticity could maintain substantial phenotypic differentiation despite very high gene flow ( $>10$ migrants per generation) and low genetic differentiation. Under maladaptive plasticity, both $P_{\mathrm{ST}}$ and $Q_{\mathrm{ST}}$ were lower than phenotypic divergence, and genetic divergence would suggest at intermediate values of gene flow, because the within-population variation $\left(G_{w}, P_{w}\right)$ was maximized under such conditions (Figure 3 and Supplementary Figure S7). High $G_{w}$ was obtained when genetically distinct individuals (due to high $D_{G}$ ) immigrate into patches at comparably high rates (high $m_{e}$ ) and contribute significant variance in additive genetic effects to a population.

\section{Comparing analytical model with simulation results}

Model predictions and simulation results agreed over most of the parameter space explored although discrepancies existed (Figure 4) when measures of population differentiation $\left(D_{P}, P_{\mathrm{ST}}, D_{G}\right.$ and $\left.Q_{\mathrm{ST}}\right)$ predicted by the analytical models were lower than those in the simulations at some parameter combinations. The discrepancies stemmed from the skewed distributions of genotypic values resulting from migration-selection balance within populations (Supplementary Figure S8) while the analytical predictions are valid under the assumption of normally distributed genotypic values, as previously shown (Yeaman and Guillaume, 2009; Débarre et al., 2015). Skewed genotypic distributions emerged when genetically distinct migrants contributed significantly to population divergence. Discrepancies were strongest for maladaptive plasticity with very negative NoR slope $\left(g_{1}<0\right.$; Figure 4$)$.

\section{Effect of plasticity on effective gene flow and genetic differentiation} $F_{\text {ST }}$ and $F_{\text {STQ }}$

Adaptive phenotypic plasticity increased the effective migration rate toward the census migration rate, by increasing the fitness of migrants toward the fitness of residents, while maladaptive plasticity decreased effective gene flow by increasing the migration load (Supplementary Figures S2a-h). Reduction of effective migration by maladaptive plasticity even permitted phenotypic divergence much closer to the optimal divergence than partially adaptive plasticity (Figures 3a and $4 \mathrm{a}$ ). By their effect on the effective migration rate, maladaptive plasticity promoted and adaptive plasticity reduced differentiation at both neutral $\left(F_{\mathrm{ST}}\right)$ and quantitative $\left(F_{\mathrm{STQ}}\right)$ loci in all three life cycles. The effect of plasticity on $F_{\mathrm{ST}}$ and $F_{\mathrm{STQ}}$ was strongest under strong selection and almost disappeared under weak selection (Figure 5, Supplementary Figure S9). In general, the largest probability to detect 

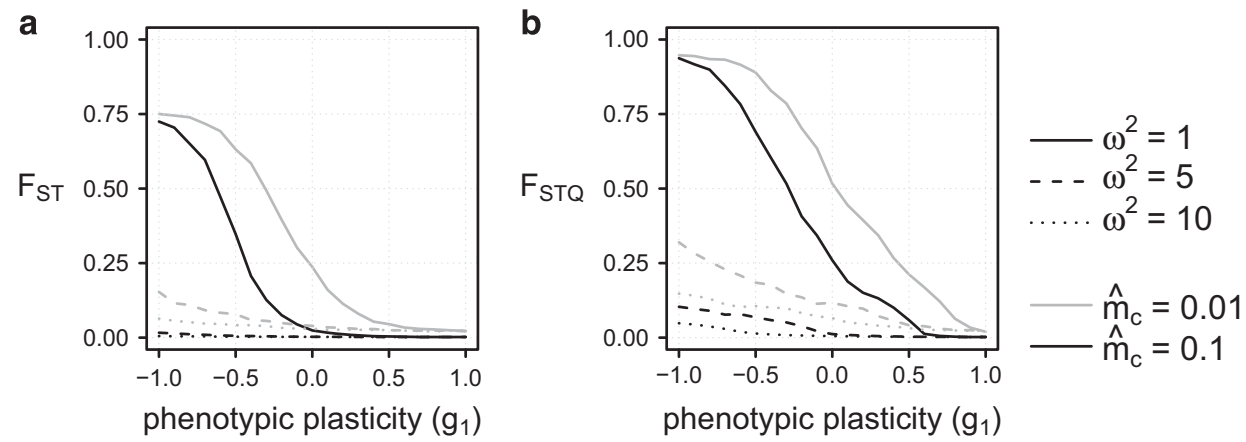

Figure 5 Simulation results for average allelic differentiation at neutral loci $F_{\mathrm{ST}}(\mathbf{a})$ and adaptive loci $F_{\mathrm{STQ}}(\mathbf{b})$ for two census migration rates $\left(\hat{m}_{c}=0.1\right.$, $\left.\hat{m}_{c}=0.01\right)$ at high and moderate selection $\left(\omega^{2}=1, \omega^{2}=5\right.$ and $\left.\omega^{2}=10\right)$ in the MS model.
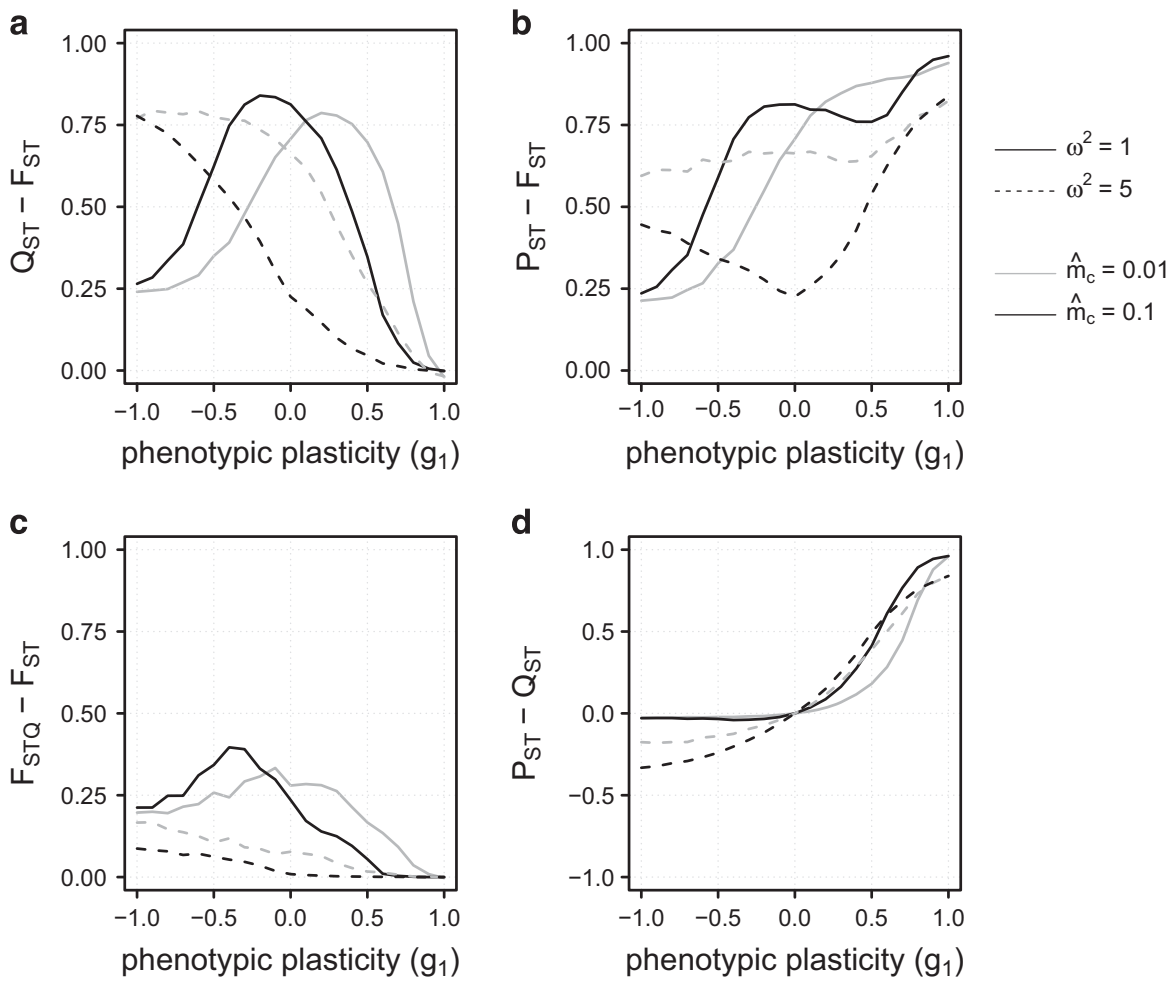

Figure 6 Simulation results for the differences $Q_{\mathrm{ST}}-F_{\mathrm{ST}}(\mathbf{a}), P_{\mathrm{ST}}-F_{\mathrm{ST}}(\mathbf{b}), F_{\mathrm{STQ}}-F_{\mathrm{ST}}(\mathbf{c})$ and $P_{\mathrm{ST}}-Q_{\mathrm{ST}}(\mathbf{d})$ for two different selection strengths $\left(\omega^{2}=1, \omega^{2}=5\right)$ as a function of the level of gene flow $\left(\hat{m}_{c}\right)$ and the degree of phenotypic plasticity $\left(g_{1}\right)$. All graphs represent the results of the MS life cycle.

loci under selection, occurring when the $F_{\mathrm{STQ}}-F_{\mathrm{ST}}$ difference is largest, was obtained under strong selection and increased with maladaptive plasticity and weaker migration (Figure 6c). Otherwise, maladaptive plasticity caused a weak increase of $F_{\mathrm{STQ}}-F_{\mathrm{ST}}$ relative to adaptive plasticity.

Consequences for the differences $Q_{\mathrm{ST}}-F_{\mathrm{ST}}, P_{\mathrm{ST}}-F_{\mathrm{ST}}$ and $F_{\mathrm{STQ}}-F_{\mathrm{ST}}$ For the differences $Q_{\mathrm{ST}}-F_{\mathrm{ST}}$ and $P_{\mathrm{ST}}-F_{\mathrm{ST}}$, adaptive plasticity generally increased $P_{\mathrm{ST}}-F_{\mathrm{ST}}$, while decreasing $Q_{\mathrm{ST}}-F_{\mathrm{ST}}$ (Figures 6a and b), in accord with the variation of $Q_{\mathrm{ST}}$ and $P_{\mathrm{ST}}$. Maladaptive plasticity showed the reverse overall tendency, with more complex interactions with migration and selection (Figures 6a and b). Adaptive differentiation $\left(Q_{S T}\right)$, however, was substantially larger at the whole-trait level $\left(Q_{\mathrm{ST}}-F_{\mathrm{ST}}\right)$ than at the locus level $\left(F_{\mathrm{STQ}}-F_{\mathrm{ST}}\right)$ when plasticity was maladaptive (compare Figures 6a and c). The differences between $Q_{\mathrm{ST}}$ and $F_{\mathrm{STQ}}$ (and thus $Q_{\mathrm{ST}}-F_{\mathrm{ST}}$ and $F_{\mathrm{STQ}}-F_{\mathrm{ST}}$ ) derived from the fact that $\mathrm{Q}_{\mathrm{ST}}$ is the result of differentiation in allele frequencies at quantitative trait locis $\left(F_{\mathrm{STQ}}\right)$ and the covariation of allelic effects within and between populations (Le Corre and Kremer, 2012). The corresponding results for genic variances and covariances are given in the Supplementary Information (Supplementary Figure S10 and S11).

\section{Life cycle effects on population differentiation}

$\mathrm{SM}$ led to less, or more negative, population divergence $\left(D_{P}\right.$, and $\left.D_{G}\right)$ at equilibrium than MS when $g_{1}<1$ (Figures 2a and b) because divergence is estimated after migration in SM but after selection has acted on maladapted immigrants in MS. In these two life cycles, phenotype determination happened in the same environment where selection was operating, erasing differences between SM and MS divergence when plasticity was adaptive. In contrast, phenotypic 
determination (development) and selection were decoupled in the DMS life cycle, which made an adaptive plastic response in the natal patch maladaptive after migration, and vice-versa for maladaptive plasticity, reversing the effect of the type of plasticity on effective migration (Supplementary Figure S2). In consequence, 'perfect' plasticity $\left(g_{1}=1\right)$ did neither necessarily cause an optimal phenotypic divergence of two (Figure 2c) nor did it always lead to a genetic divergence of zero (Figure 2d). Instead, optimal phenotypic divergence was achieved at a lower degree of plasticity $\left(g_{1}=1-G_{w} /\left(\omega^{2}+P_{w}\right)\right)$. The difference between DMS and MS predictions increased with migration and the slope of the NoR (Figure 2), although it remained low.

\section{DISCUSSION}

Different populations often experience different environmental conditions, which requires the evolution of different phenotypes to maximize fitness. As a consequence, divergent selection can lead to genetic and phenotypic variation within a species' range. Gene flow between populations, however, counteracts divergent selection and causes genetic homogenization. In such situations of divergent selection with gene flow, population differentiation may be strongly affected by phenotypic plasticity (Crispo, 2008). By extending a quantitative genetics model (Hendry et al., 2001) and using individual-based simulations, we investigated the effect of phenotypic plasticity on genetic and phenotypic differentiation and divergence between two populations. Population differentiation, either genetic or phenotypic, of traits under selection is often wishfully interpreted as causally linked to adaptive fitness differentiation across space. We show that phenotypic plasticity breaks the correspondence between genetic or phenotypic differentiation and local adaptation. In particular, adaptive phenotypic plasticity promotes phenotypic adaptive differences while canceling local adaptation by selecting for genetic similarity across space and enhancing homogenization by gene flow. A given genotype would here have similar fitness across environments (for example, when doing a transplant experiment) despite phenotypic differences, thus providing evidence of absence of local adaptation and presence of adaptive phenotypic plasticity. As the plastic response becomes more adaptive, genetic differentiation slowly vanishes and gene flow may falsely appear as not constraining local adaptation. Maladaptive phenotypic plasticity has the opposite effect as it increases genetic divergence between populations while not being able to maintain as much phenotypic differentiation as without plasticity. However, maladaptive plasticity can promote phenotypic divergence when it reduces the effective migration rate and thereby allows for high genetic compensation. It can also cause populations to diverge phenotypically in a direction opposite to the environmental or genetic divergence, thus generating countergradient variation.

\section{On the interaction between phenotypic plasticity and gene flow}

From our mathematical model, phenotypic divergence between the two populations depends on an interaction between migration and plasticity (the product of $\hat{m}$ and $g_{1}$, Equations (6), (A3) and (A9)), while genetic divergence is a monotonic function of both parameters (Equation (7), but see Equations (A4) and (A8)). Our individual-based simulations corroborate these predictions but further show how plasticity modulates gene flow. The analytical model considers the $\hat{m}$ parameter, which corresponds to the effective rate of immigration of individuals or gametes from one to the other population. Instead, our simulations show that the effective immigration of individuals into a patch depends not only on the census migration rate but also on the effect of plasticity on selection, as has been described conceptually by Crispo (2008). Under adaptive plasticity, the effective migration rate is close to the rate of census migration because plasticity mitigates the effect of divergent selection by allowing the expression of the adaptive phenotype irrespective of the origin of the individual (Thibert-Plante and Hendry, 2011). In contrast, maladaptive plasticity reduces the effective immigration rate because it leads to the expression of even more maladapted phenotypes in the foreign environment, allowing selection to quickly remove maladapted immigrants and thereby augmenting isolation by adaptation (Nosil et al., 2008; Orsini et al., 2013). As a result, at intermediate census migration, phenotypic divergence increases with maladaptive plasticity, even allowing for larger divergence than with partially adaptive plasticity.

\section{Implications of phenotypic plasticity shaping $P_{\mathrm{ST}}$ and $Q_{\mathrm{ST}}$}

Although it is generally understood that $P_{\mathrm{ST}}$ is a bad surrogate for $Q_{\mathrm{ST}}$ in $Q_{\mathrm{ST}}-F_{\mathrm{ST}}$ comparisons (Falconer and Mackay, 1996; Pujol et al., 2008; Brommer, 2011), understanding the relationship between $P_{\mathrm{ST}}$ and $Q_{\mathrm{ST}}$ can help us understand how plasticity affects population differentiation in the wild. Our models allow to take a closer look at this important question. In general, our results suggest that plasticity will always induce a difference between $P_{\mathrm{ST}}$ and $Q_{\mathrm{ST}}$ because of the difference between the phenotypic and genetic population divergences it creates (for example, Figure 6d). Adaptive plasticity should then cause a positive $P_{\mathrm{ST}}-Q_{\mathrm{ST}}$ difference, while maladaptive plasticity should cause a negative difference, as seen in our simulations (Supplementary Figure S12). Maladaptive plasticity can also lead to shallow genetic gradients $\left(Q_{\mathrm{ST}} \sim=0\right)$ with large phenotypic differentiation $\left(P_{\mathrm{ST}}>0\right)$ when high migration constrains genetic divergence and plasticity is the main contributor to trait divergence (for example, see Figures $3 \mathrm{~b}$ and $\mathrm{d}$ when $m_{c} \sim 0.2-0.3$; Supplementary Figure S12). Isoclines of $P_{\mathrm{ST}}=Q_{\mathrm{ST}}$ may thus appear even when $g_{1} \neq 0$ under specific parameter values for $g_{1}<0$ and large migration rates, rendering the interpretation of $P_{\mathrm{ST}}-Q_{\mathrm{ST}}$ cumbersome. Moreover, these general patterns come with many caveats caused by factors that we did not consider in our models. For instance, a positive difference, interpreted as evidence for adaptive plasticity, may also be reached when plasticity is much higher than optimal plasticity (maladaptive hyperplasticity), a situation that we have not covered in our simulations. Furthermore, a negative $P_{\mathrm{ST}}-Q_{\mathrm{ST}}$ difference can be reached even in the absence of plasticity simply because the within-population component of phenotypic variance $\left(P_{w}\right)$ is often affected by nonadditive and environmental effects, and it is thus expected to be larger than its genetic homolog $\left(G_{w}\right)$. Both $P_{\mathrm{ST}}$ and $Q_{\mathrm{ST}}$ are ratios of two variance components that can deviate from each other differently whether at the phenotypic or genetic level and lead to the presence or absence of differences between $P_{\mathrm{ST}}$ and $Q_{\mathrm{ST}}$. Therefore, we should be cautious when interpreting such differences and always look at the underlying differences between the phenotypic and genetic variance components from which we can gain better insights into the causes of phenotypic differentiation.

\section{Conclusions derived from life cycle comparisons}

The effects of phenotypic plasticity on the ability of a species to adapt to heterogeneous environments is expected to depend on the timing of development relative to migration and selection (De Jong and Behera, 2002; Thibert-Plante and Hendry, 2011; Scheiner et al., 2012). Typically, the adaptive value of plasticity depends on whether development happens in the environment of selection or not. If not, like in the DMS life cycle, the adaptivity of the plastic response of the genotype is reversed, but for the migrating individuals only, making an 
adaptive plastic response in the home patch maladaptive in the foreign patch, and vice-versa for maladaptive plasticity. Yet, plasticity in DMS increases the adaptive phenotypic divergence when adaptive, and makes it more negative when maladaptive (Figure 2), instead of reducing the adaptive or maladaptive divergence, as might be expected. This result can be understood as follows. In DMS, selection within population becomes stronger under adaptive plasticity because of the larger phenotypic effect of the maladapted immigrants, which results in smaller effective gene flow but also in an increased response to selection. That response actually leads to a genetic compensation within populations, favoring more extreme genotypes, to the point of overshooting the optimum phenotype, and hence the optimum phenotypic divergence too (Figure 2). In contrast, selection becomes weaker under maladaptive plasticity and leads to increased introgression of the maladapted genotypes. Because those genotypes are actually farther away from the optimum trait value and from the population mean, populations then express a larger maladaptive plastic response which steepens the phenotypic cline between the two populations. Finally, the difference between MS and SM is more intuitive because development always takes place in the same patch as selection. MS and SM only differ in the point in time where divergence is measured: after dispersal in SM (a process reducing divergence) and after selection in MS (which increases divergence). Yet, that difference also has biological meaning because in some species development, and selection, may happen at the juvenile stage prior to migration (for example, bird or animal species), or after migration when juveniles develop in the new environment (for example, after seed dispersal).

Other simulation studies similarly emphasized the interaction between life cycle characteristics and adaptive phenotypic plasticity, but mostly ignore the effects of maladaptive plasticity. For example, Scheiner and Holt (2012) and Thibert-Plante and Hendry (2011) showed for divergent selection in structured populations that the evolved levels of adaptive plasticity were lower with a DMS than with a SM life cycle, as adaptive plasticity was not necessarily beneficial for migrants in the selecting environment. Furthermore, adaptive plasticity has been shown to favor the evolution of reproductive barriers between diverging habitats when in combination with evolving assortative mating (Thibert-Plante and Hendry, 2011), adaptive habitat choice (Nonaka et al., 2015) or evolving dispersal rates (Scheiner et al., 2012). These results, in combination with ours, emphasize that phenotypic plasticity and a species' ecology may have deep consequences for the course of evolution in heterogeneous environments (Pelletier et al., 2009; Ghalambor et al., 2015; Hendry, 2016). Approaches that aim to predict evolutionary dynamics (for example, in response to climate change) therefore should account for species-specific migration rates, trait-specific plastic responses and life cycle characteristics (Guisan and Thuiller, 2005; Schiffers et al., 2013; Cotto et al., 2017).

\section{Maladaptive plasticity, countergradient variation and the extent of genetic evolution \\ Although maladaptive plasticity is commonly ignored in theoretical studies, ample empirical evidence exists for its consequences on phenotypic variation across environmental gradients-countergradient variation in phenotypic traits. Countergradient variation describes genetic differences opposed to the plastic response and can either be based on hyperplasticity or maladaptive plasticity (Levins, 1969; Conover and Present, 1990; Conover et al., 2009). A typical case is obtained when phenotypic clines are much shallower than genetic clines (Craig and Foote, 2001; Grether, 2005; Jiménez-Ambriz et al.,}

2007; Deere et al., 2012). It happens in our model with maladaptive plasticity when a critical value of the NoR is reached $\left(g_{1}=-G / \hat{m}\left(w^{2}+P-G\right)\right.$; from Equations (6) and (7)) at which $D_{P}=0$ but $D_{G}>0$. Many examples have been described in more than 60 plant, fish and insect species (Conover et al., 2009). Recent empirical work further highlighted that maladaptive plasticity can 'potentiate' genetic adaptation by accentuating the effects of directional selection on plastic gene expression levels of adaptive loci (Dayan et al., 2015; Ghalambor et al., 2015; Healy and Schulte, 2015). Ghalambor et al. (2015) reached this conclusion by studying the early stage of adaptation of Trinidadian guppies to a new predator-free environment. They found that among the 135 transcripts that showed signs of genetic adaptive change in their level of expression, the majority (89\%) exhibited maladaptive plasticity prior to adaptation, while transcripts with adaptive plasticity showed reduced genetic divergence between populations. Our results are in complete agreement with these empirical findings (see also Dayan et al., 2015), although we focused on spatial differences in the environment with gene flow and did not attempt to model variation of plasticity among loci.

What are then the evolutionary consequences of maladaptive plasticity? The fact that plasticity can at the same time 'potentiate' adaptation and be maladaptive seems contradictory. It does indeed increase selection on the genotypes within a population and thus facilitates genetic change and divergence. But, being maladaptive, it also decreases the population average fitness by its effects on the phenotypes, to the point of sometimes causing population extinction when selection is least efficient against maladaptive gene flow (for example, with pollen dispersal, as in the SM model) and selection is strong. We can thus expect detrimental effects of maladaptive plasticity on the demography and evolutionary dynamics of a population. Countergradient variation can therefore be a sign of a reduced ability of a species to adapt to heterogeneous environments, particularly in the context of temporally varying environments (for example, climate change). Using Chevin et al. (2010) framework, it can be shown that maladaptive plasticity reduces the maximum tolerable rate of environmental change. It would also reduce a species' capacity of establishment in new habitats and, in conjunction with its adverse effects on migrants selecting against dispersal, can be expected to generally reduce the capacity of a species to expand its range. However, scenarios of countergradient variation and maladaptive plasticity might also represent situations with high magnitudes of genetic evolution (Dayan et al., 2015; Ghalambor et al., 2015) and thus high potential for evolutionary rescue if the negative demographic consequences are overcome. More work is necessary to delineate the multiple effects of maladaptive plasticity on the evolution of a species' range.

\section{Limitations}

In any case, using our modification of the Hendry et al. (2001) model to infer evolutionary parameters in empirical studies can be cautiously done taking account of a few caveats. Almost all natural systems should deviate from the perfectly symmetric two-patch system modeled. Therefore, it could be interesting to extend the models to more complex scenarios with population-specific variances in environmental conditions, selection strengths or asymmetric gene flow. Also, the average NoR slopes of the two populations might not be parallel when benefits, limitations or costs of phenotypic plasticity differ between populations. Our model is designed for divergent selection when different phenotypes are favored in different environments. However, situations have been reported when the same 
phenotype is favored in different environments while the different environments cause a different plastic response (Craig and Foote, 2001; Grether, 2005). For example, female Trinidadian guppies (Poecilia reticulata) prefer intermediate ratios between carotenoid and drosopterin pigments in orange spots of males. However, differences in the diet can lead to deviations in male coloration from optimal levels, which led to genetic compensation and constant pigment ratios across environments (Deere et al., 2012). To account for these situations, our model requires a slight modification such that the difference in the environment $e_{j}-e_{i}$ is not equated with the difference in phenotypic optima $\theta_{j}-\theta_{i}$.

\section{DATA ARCHIVING}

The Nemo source code, Nemo init files and summary of simulation results are available from the Dryad Digital Repository: http://dx.doi. org/10.5061/dryad.418p5.

\section{CONFLICT OF INTEREST}

The authors declare no conflict of interest.

\section{ACKNOWLEDGEMENTS}

We thank Cortland Griswold, Sam Scheiner, Andrew Hendry, Stephen Proulx, Axios Review and two anonymous referees for their valuable comments on the manuscript and constructive discussions. Simulations were run on the UZH Science Cloud. MS and FG were financed by an SNF grant (PP00P3_144846).

Ahlgren J, Yang X, Hansson L, Brönmark C (2013). Camouflaged or tanned: plasticity in freshwater snail pigmentation. Biol Lett 9: 20130464.

Beldade P, Mateus ARA, Keller RA (2011). Evolution and molecular mechanisms of adaptive developmental plasticity. Mol Ecol 20: 1347-1363.

Blanquart F, Gandon S, Nuismer SL (2012). The effects of migration and drift on local adaptation to a heterogeneous environment. J Evol Biol 25: 1351-1363.

Bradshaw AD (1965). Evolutionary significance of phenotypic plasticity in plants. Adv Genet 13: 115-155.

Brommer JE (2011). Whither $P_{S T}$ ? The approximation of $Q_{S T}$ by $P_{S T}$ in evolutionary and conservation biology. J Evol Biol 24: 1160-1168.

Chevin L-M, Lande R (2011). Adaptation to marginal habitats by evolution of increased phenotypic plasticity. J Evol Biol 24: 1462-1476.

Chevin L-M, Lande R (2015). Evolution of environmental cues for phenotypic plasticity. Evolution 69: 2767-2775.

Chevin L-M, Lande R, Mace GM (2010). Adaptation, plasticity, and extinction in a changing environment: towards a predictive theory. PLoS Biol 8: e1000357.

Conover DO, Duffy TA, Hice LA (2009). The covariance between genetic and environmental influences across ecological gradients: Reassessing the evolutionary significance of countergradient and cogradient variation. Annal N Y Acad Sci 1168 $100-129$.

Conover DO, Present TMC (1990). Countergradient variation in growth rate : compensation for length of the growing season among Atlantic Silversides from different latitudes. Oecologia 83: 316-324.

Conover DO, Schultz ET (1995). Phenotypic similarity and the evolutionary significance of countergradient variation. Trends Ecol Evol 10: 248-252.

Le Corre V, Kremer A (2012). The genetic differentiation at quantitative trait loci under local adaptation. Mol Ecol 21: 1548-1566.

Cotto O, Wessely J, Georges D, Klonner G, Schmid M, Dullinger S et al. (2017). A dynamic eco-evolutionary model predicts slow response of alpine plants to climate warming. $\mathrm{Nat}$ Commun 8: 15399.

Craig JK, Foote CJ (2001). Countergradient variation and secondary sexual color: phenotypic convergence promotes genetic divergence in carotenoid use between sympatric anadromous and nonanadromous morphs of sockeye salmon (Oncorhynchus nerka). Evolution 55: 380-391.

Crispo $E$ (2008). Modifying effects of phenotypic plasticity on interactions among natural selection, adaptation and gene flow. J Evol Biol 21: 1460-1469.

Dayan DI, Crawford DL, Oleksiak MF (2015). Phenotypic plasticity in gene expression contributes to divergence of locally adapted populations of Fundulus heteroclitus. Mol Ecol 24: 3345-3359.

Débarre F, Yeaman S, Guillaume F (2015). Evolution of quantitative traits under a migration-selection balance: when does skew matter? Am Nat 186: S37-S47.

Deere KA, Grether GF, Sun A, Sinsheimer JS (2012). Female mate preference explains countergradient variation in the sexual coloration of guppies (Poecilia reticulata). Proc $R$ Soc B Biol Sci 279: 1684-1690.
Falconer DS, Mackay TFC (1996). Introduction to Quantitative Genetics, 4th edn. Pearson Prentice Hall: Harlow, Essex, UK.

García-Ramos G, Kirkpatrick M (1997). Genetic models of adaptation and gene glow in peripheral populations. Evolution 51: 21-28.

Gavrilets S, Scheiner SM (1993). The genetics of phenotypic plasticity. VI. Theoretical predictions for directional selection. J Evol Biol 6: 49-68.

Ghalambor CK, Hoke KL, Ruell EW, Fischer EK, Reznick DN, Hughes KA (2015). Nonadaptive plasticity potentiates rapid adaptive evolution of gene expression in nature. Nature 525: 372-375.

Ghalambor CK, McKay JK, Carroll SP, Reznick DN (2007). Adaptive versus non-adaptive phenotypic plasticity and the potential for contemporary adaptation in new environments. Funct Ecol 21: 394-407.

Goudet J (2005). HIERFSTAT, a package for $\mathrm{R}$ to compute and test hierarchical F-statistics. Mol Ecol Notes 5: 184-186.

Grether GF (2005). Environmental change, phenotypic plasticity, and genetic compensation. Am Nat 166: E115-E123.

Guillaume F, Rougemont J (2006). Nemo: An evolutionary and population genetics programming framework. Bioinformatics 22: 2556-2557.

Guisan A, Thuiller W (2005). Predicting species distribution: offering more than simple habitat models. Ecol Lett 8: 993-1009.

Healy TM, Schulte PM (2015). Phenotypic plasticity and divergence in gene expression. Mol Ecol 24: 3220-3222.

Hendry AP (2016). Key questions on the role of phenotypic plasticity in eco-evolutionary dynamics. J Hered 107: 25-41.

Hendry AP, Day T, Taylor EB (2001). Population mixing and the adaptive divergence of quantitative traits in discrete populations: a theoretical framework for empirical tests. Evolution 55: 459-466.

Huisman J, Tufto J (2012). Comparison of non-gaussian quantitative genetic models for migration and stabilizing selection. Evolution 66: 3444-3461.

Jiménez-Ambriz G, Petit C, Bourrié I, Dubois S, Olivieri I, Ronce O (2007). Life history variation in the heavy metal tolerant plant Thlaspi caerulescens growing in a network of contaminated and noncontaminated sites in southern France: role of gene flow, selection and phenotypic plasticity. New Phytol 173: 199-215.

Johannsen W (1911). The genotype conception of heredity. Am Nat 45: 129-159.

De Jong G (1990). Genotype-by-environment interaction and the genetic covariance between environments: Multilocus genetics. Genetica 81: 171-177.

De Jong G, Behera N (2002). The influence of life-history differences on the evolution of reaction norms. Evol Ecol Res 4: 1-25.

Kirkpatrick M, Barton NH (1997). Evolution of a species' range. Am Nat 150: 1-23.

Lande R (2009). Adaptation to an extraordinary environment by evolution of phenotypic plasticity and genetic assimilation. J Evol Biol 22: 1435-1446.

Lande R (2014). Evolution of phenotypic plasticity and environmental tolerance of a labile quantitative character in a fluctuating environment. J Evol Biol 27: 866-875.

Langerhans RB, DeWitt TJ (2002). Plasticity constrained: over-generalized induction cues cause maladaptive phenotypes. Evol Ecol Res 4: 857-870.

Leinonen T, Cano JM, Mäkinen H, Merilä J (2006). Contrasting patterns of body shape and neutral genetic divergence in marine and lake populations of threespine sticklebacks. J Evol Biol 19: 1803-1812.

Lenormand T (2002). Gene flow and the limits to natural selection. Trends Ecol Evol 17: 183-189.

Levins R (1969). Thermal acclimation and heat resistance in Drosophila species. Am Nat 103: 483-499.

Lewontin RC, Krakauer J (1973). Distribution of gene frequency as a test of the theory of the selective neutrality of polymorphisms. Genetics 74: 175-195.

Merilä J, Laurila A, Laugen AT, Räsänen K, Pahkala M (2000). Age and size at metamorphosis in Rana temporaria: comparison of high and low latitude populations. Ecography 23: 457-465.

Nonaka E, Svanbäck R, Thibert-Plante X, Englund G, Brännström Å (2015). Mechanisms by which phenotypic plasticity affects adaptive divergence and ecological speciation. Am Nat 186: E126-E143.

Nosil P, Egan SP, Funk DJ (2008). Heterogeneous genomic differentiation between walking-stick ecotypes: 'Isolation by adaptation' and multiple roles for divergent selection. Evolution 62: 316-336.

Orsini L, Vanoverbeke J, Swillen I, Mergeay J, De Meester L (2013). Drivers of population genetic differentiation in the wild: Isolation by dispersal limitation, isolation by adaptation and isolation by colonization. $\mathrm{Mol}$ Ecol 22: 5983-5999.

Pelletier F, Garant D, Hendry APP (2009). Eco-evolutionary dynamics. Philos Trans $R$ Soc B: Biol Sci 364: 1483-1489.

Pujol B, Wilson AJ, Ross RIC, Pannell JR (2008). Are QST $-F_{S T}$ comparisons for natural populations meaningful? Mol Ecol 17: 4782-4785.

R Development Core Team (2008). R: a language and environment for statistical computing.

Rellstab C, Gugerli F, Eckert AJ, Hancock AM, Holderegger R (2015). A practical guide to environmental association analysis in landscape genomics. Mol Ecol 24: 4348-4370.

Scheiner SM (1993). Genetics and evolution of phenotypic plasticity. Annu Rev Ecol Syst 24: 35-68.

Scheiner SM (2013). The genetics of phenotypic plasticity. XII. Temporal and spatial heterogeneity. Ecol Evol 3: 4596-4609.

Scheiner SM, Barfield M, Holt RD (2012). The genetics of phenotypic plasticity. XI. Joint evolution of plasticity and dispersal rate. Ecol Evol 2: 2027-2039.

Scheiner SM, Holt RD (2012). The genetics of phenotypic plasticity. X. Variation versus uncertainty. Ecol Evol 2: 751-767. 
Schiffers K, Bourne EC, Lavergne S, Thuiller W, Travis JMJ (2013). Limited evolutionary rescue of locally adapted populations facing climate change. Philos Trans $R$ Soc Lond $B$ Biol Sci 368: 20120083.

Schlichting CD, Pigliucci M (1998). Phenotypic Evolution. A Reaction Norm Perspective. Sinauer Associates, Inc.: Sunderland, MA, USA.

Seehausen O, Butlin RK, Keller I, Wagner CE, Boughman JW, Hohenlohe PA et al. (2014). Genomics and the origin of species. Nat Rev Genet 15: 176-192.

Slatkin M (1987). Gene flow and the geographic structure of natural populations. Science 236: 787-792.

Spitze K (1993). Population structure in Daphnia obtusa: quantitative genetic and allozymic variation. Genetics 135: 367-374.

Stearns SC (1989). The evolutionary significance of phenotypic plasticity. BioScience 39: 436-445.

Storz JF (2002). Contrasting patterns of divergence in quantitative traits and neutral DNA markers: Analysis in clinal variation. Mol Ecol 11: 2537-2551.

Sultan SE, Spencer HG (2002). Metapopulation structure favors plasticity over local adaptation. Am Nat 160: 271-283.
Thibert-Plante X, Hendry AP (2011). The consequences of phenotypic plasticity for ecological speciation. J Evol Biol 24: 326-342.

Via S, Lande R (1985). Genotype-environment interaction and the evolution of phenotypic plasticity. Evolution 39: 505-522.

West-Eberhard MJ (1989). Phenotypic plasticity and the origins of diversity. Annu Rev Ecol Syst 20: 249-278.

Whitlock MC (2008). Evolutionary inference from QST. Mol Ecol 17: 1885-1896.

Whitlock MC, Lotterhos KE (2015). Reliable detection of loci responsible for local adaptation: inference of a null model through trimming the distribution of $\mathrm{F}_{\mathrm{ST}}$. Am Nat 186: S24-S36.

Woltereck R (1909). Weitere experimentelle Untersuchungen über Artveränderung, speziell über das Wesen quantitativer Artunterschiede bei Daphnien. Verh Dtsch Zool Ges 19: $110-173$.

Yeaman S, Guillaume F (2009). Predicting adaptation under migration load: The role of genetic skew. Evolution 63: 2926-2938.

Supplementary Information accompanies this paper on Heredity website (http://www.nature.com/hdy)

\section{APPENDIX}

\section{Phenotypic and genetic divergence (Selection-Migration)}

In the second model, selection acts before migration. Again, the phenotype of migrants is expressed in dependence of the same environment as selection occurs. Extending the original equations from Hendry et al. (2001), the trait values after selection, reproduction and then migration $\left(\bar{z}_{i}^{s m}, \bar{z}_{j}^{s m}\right)$ are calculated as follows.

$$
\begin{aligned}
\bar{z}_{i}^{s m}= & m_{i}\left(\bar{g}_{0 j}+\bar{g}_{1} e_{i}+G_{w j} \beta_{j}\left(\bar{z}_{j}\right)\right) \\
& +\left(1-m_{i}\right)\left(\bar{g}_{0 i}+\bar{g}_{1} e_{i}+G_{w i} \beta_{i}\left(\bar{z}_{i}\right)\right) \\
\bar{z}_{j}^{s m}= & m_{j}\left(\bar{g}_{0 i}+\bar{g}_{1} e_{j}+G_{w i} \beta_{i}\left(\bar{z}_{i}\right)\right) \\
& +\left(1-m_{j}\right)\left(\bar{g}_{0 j}+\bar{g}_{1} e_{j}+G_{w j} \beta_{j}\left(\bar{z}_{j}\right)\right)
\end{aligned}
$$

Phenotypic plasticity refers to labile and developmental phenotypic plasticity when selection occurs after phenotype expression before dispersal (the selection gradient $\beta$ acts on $z_{j}$ and $z_{i}$ before dispersal). The calculations of $\Delta \bar{z}=\bar{z}^{s m}-\bar{z}$ and $\Delta D=\Delta \bar{z}_{j}-\Delta \bar{z}_{i}$ allow to compute the mean difference in trait value $\left(D_{P}^{*}\right)$ after selection and then migration at equilibrium $(\Delta D=0)$.

$$
D_{P_{s m}}^{*}=\bar{g}_{1}\left(e_{j}-e_{i}\right)+\left(\frac{1-\hat{m}}{\hat{m}}\right)\left[G_{w j} \beta_{j}\left(\bar{z}_{j}\right)-G_{w i} \beta_{i}\left(\bar{z}_{i}\right)\right]
$$

Together with a Gaussian selection gradient and the assumption of equal phenotypic and genetic variances in both populations $\left(P_{w}=P_{w i}=P_{w j} ; G_{w}=G_{w i}=G_{w j}\right)$, the difference in mean trait values between populations in dependence of gene flow and phenotypic plasticity is derived.

$$
D_{P_{s m}}^{*}=D_{\Theta} \frac{G_{w}(1-\hat{m})+g_{1} \hat{m}\left(\varpi^{2}+P_{w}\right)}{G_{w}+\hat{m}\left(\varpi^{2}+P_{w}-G_{w}\right)}
$$

Again, the change in the trait value of individuals dispersing from population $i$ to $j$ as result of phenotypic plasticity is referred to as $D_{E}=g_{1}\left(e_{j}-e_{i}\right)=g_{1} D_{\Theta}$. Similar to Equation (7), the genetic divergence $\left(D_{G s m}^{*}\right)$ can be calculated from $D_{P_{s m}}^{*}$ and $D_{E}$.

$$
D_{G s m}^{*}=D_{\Theta} \frac{G_{w}(1-\hat{m})\left(1-g_{1}\right)}{G_{w}+\hat{m}\left(\varpi^{2}+P_{w}-G_{w}\right)}
$$

\section{Phenotypic and genetic divergence (Development-Migration- Selection)}

In the third model, the phenotype is expressed before migration and subsequent selection. In contrast to the previous two life cycles, it is easier to calculate genetic divergence (the difference between the NoR intercept values) before phenotypic divergence. The intercept values after development, migration, selection and reproduction $\left(\bar{z}_{i}^{d m s}, \bar{z}_{j}^{d m s}\right)$ are calculated as follows.

$$
\begin{aligned}
& \bar{g}_{0 i}^{d m s}=m_{i}\left(\bar{g}_{0 j}\right)+\left(1-m_{i}\right)\left(\bar{g}_{0 i}\right)+G_{w i} \beta_{i}\left(\bar{z}_{i}^{m}\right) \\
& \bar{g}_{0 j}^{d m s}=m_{j}\left(\bar{g}_{0 i}\right)+\left(1-m_{j}\right)\left(\bar{g}_{0 j}\right)+G_{w j} \beta_{j}\left(\bar{z}_{j}^{m}\right)
\end{aligned}
$$

Phenotypic plasticity refers to labile and developmental phenotypic plasticity when the phenotype expression is realized before migration and subsequent selection (that is, the selection gradient $\beta$ acts on the phenotypes $z_{j}^{m}$ and $z_{i}^{m}$ after phenotype expression in the home environment and then dispersal). The calculations of $\Delta \bar{g}=\bar{g}^{d m s}-\bar{g}$ then allow to derive $\Delta D_{G}=\Delta \bar{g}_{0 \mathrm{j}}-\Delta \bar{g}_{0 \mathrm{i}}$ as

$$
\Delta D_{G}=\left(m_{j}+m_{i}\right)\left(g_{0 i}-g_{0 j}\right)+G_{w j} \beta_{j}\left(\bar{z}_{j}^{m}\right)-G_{w i} \beta_{i}\left(\bar{z}_{i}^{m}\right)
$$

At equilibrium $(\Delta D=0)$, the mean genetic divergence $\left(D_{G}^{*}\right)$ after development, migration and selection then is

$$
D_{G d m s}^{*}=\left(\frac{1}{\hat{m}}\right)\left[G_{w j} \beta_{j}\left(\bar{z}_{j}^{m}\right)-G_{w i} \beta_{i}\left(\bar{z}_{i}^{m}\right)\right]
$$

Using a Gaussian selection gradient $\left(\beta(z)=-(z-\Theta) /\left(\omega^{2}+P_{w}\right)\right)$ with $\bar{z}_{j}^{m}=m_{j}\left(\bar{g}_{0 i}+g_{1} e_{i}\right)+\left(1-m_{j}\right)\left(\bar{g}_{0 j}+g_{1} e_{j}\right)$ and $\bar{z}_{i}^{m}=$ $m_{i}\left(\bar{g}_{0 j}+g_{1} e_{j}\right)+\left(1-m_{i}\right)\left(\bar{g}_{0 i}+g_{1} e_{i}\right)$ as well as assuming equal phenotypic and genetic variances in both populations $\left(P_{w}=P_{w i}=P_{w j}\right.$; $\left.G_{w}=G_{w i}=G_{w j}\right)$, the average genetic divergence between populations at equilibrium can be derived.

$$
D_{G d m s}^{*}=D_{\Theta} \frac{G_{w}\left(1-g_{1}(1-\hat{m})\right)}{G_{w}+\hat{m}\left(\varpi^{2}+P_{w}-G_{w}\right)}
$$

Again, the phenotypic difference as result of phenotypic plasticity is referred to as $D_{E}=g_{1}\left(e_{j}-e_{i}\right)=g_{1} D_{\Theta}$ and $\hat{m}=m_{i}+m_{j}$ refers to the total effective migration rate. Phenotypic divergence $\left(D_{P d m s}^{*}\right)$ can be calculated from $D_{G d m s}^{*}$ and $D_{E}$ by $D_{P}=D_{G}+g_{1}\left(e_{j}-e_{i}\right)$.

$$
D_{P d m s}^{*}=D_{\Theta} \frac{\left(G_{w}+g_{1} \hat{m}\left(\omega^{2}+P_{w}\right)\right)}{G_{w}+\hat{m}\left(\varpi^{2}+P_{w}-G_{w}\right)}
$$


Note that $D_{P}$ in this life cycle corresponds to phenotypic divergence after development before migration when the phenotypes present within a population are exclusively expressed in dependence of the respective environmental condition. In the absence of phenotypic plasticity $\left(g_{1}=0\right)$, Equations (A8) and (A9) reduce to the original Migration-Selection model of Hendry et al. (2001). Perfect phenotypic divergence $\left(D_{P}=D_{\Theta}\right)$ is not achieved at $g_{1}=1$ (that is, the NoR slope connecting the two phenotypic optima), but at a lower degree of plasticity $g_{1}=1-G_{w} /\left(\omega^{2}\right.$ $\left.+P_{w}\right)$ when there is gene flow between populations $(\hat{m}>0)$. At perfect phenotypic divergence $\left(D_{P}=D_{\Theta}\right)$, the genetic divergence is $D_{G}=D_{\Theta}$ $G_{w} /\left(\omega^{2}+P_{w}\right)$ and thus not zero as long as the populations differ in their phenotypic optima $\left(D_{\Theta} \neq 0\right)$ and additive genetic variance is present $\left(G_{w}>0\right)$. Genetic divergence becomes zero at $g_{1}=1 /(1-\hat{m})$, and thereby not at perfect plasticity when gene flow is present. 